\title{
Cranial-to-caudal approach for radical lymph node dissection along the surgical trunk in laparoscopic right hemicolectomy
}

\author{
Takeru Matsuda $\cdot$ Takeshi Iwasaki $\cdot$ Masaaki Mitsutsuji • \\ Kenro Hirata $\cdot$ Yoko Maekawa $\cdot$ Tomoko Tanaka • \\ Etsuji Shimada $\cdot$ Yoshihiro Kakeji
}

Received: 4 January 2014/ Accepted: 7 July 2014/Published online: 19 August 2014

(C) Springer Science+Business Media New York 2014

\begin{abstract}
Complete mesocolic excision with central vascular ligation is considered to contribute to superior oncological outcomes after colon cancer surgery [1]. For advanced right-sided colon cancer, this surgery sometimes requires lymph node $(\mathrm{LN})$ dissection along the superior mesenteric vein (SMV), with division of the middle colic vessels, or their right branches, at origin [2]. Here, we present cranially approached radical LN dissection along the surgical trunk during laparoscopic right hemicolectomy. The omental bursa is first opened wide, and the gastrocolic trunk of Henle is exposed, using the right gastroepiploic vessels and the accessory right colic vein (ARCV) as landmarks. After division of ARCV, SMV and middle colic vein $(\mathrm{MCV})$ are identified. After dividing $\mathrm{MCV}$ at its root, $\mathrm{LN}$ dissection along SMV is conducted in a cranial-to-caudal manner. Concurrently, the middle colic artery, or its right branch, is exposed and divided at origin. The transverse colon is then raised ventrally, and LN dissection along SMV using a cranial-to-caudal approach is again performed. The ileocolic and right colic vessels are divided at origin. The ascending and transverse mesocolon, including the pedicles, are then separated from the
\end{abstract}

Electronic supplementary material The online version of this article (doi:10.1007/s00464-014-3761-x) contains supplementary material, which is available to authorized users.

T. Matsuda $(\bowtie) \cdot$ T. Iwasaki · M. Mitsutsuji - K. Hirata ·

Y. Maekawa $\cdot$ T. Tanaka $\cdot$ E. Shimada

Department of Surgery, National Hospital Organization Kobe

Medical Center, 3-1-1 Nishi-Ochiai, Suma-Ku, Kobe 654-0155,

Japan

e-mail: takerumatsuda@nifty.com

Y. Kakeji

Division of Gastrointestinal Surgery, Department of Surgery, Kobe University Graduate School of Medicine, Kobe, Japan retroperitoneal tissues, pancreatic head, and duodenum, using a medial approach. The key characteristics in this procedure consist of easy access to pancreas, early division of ARCV and middle colic vessels at origin, and easy dissection along SMV. We performed a laparoscopic colectomy using this approach for 18 patients with rightsided colon cancer. The mean operative time and blood loss were $288 \mathrm{~min}$ and $83 \mathrm{ml}$, respectively. The mean number of harvested LNs was 24 . There were 6 cases with positive $\mathrm{LN}$ metastasis. There were no recurrent cases at a median follow-up period of 24 months. We consider this approach to be safe and useful for radical LN dissection along SMV for right-sided colon cancers.

Keywords Colon cancer · Right hemicolectomy · Laparoscopy $\cdot$ Cranial approach

Disclosures Takeru Matsuda, Takeshi Iwasaki, Masaaki Mitsutsuji, Kenro Hirata, Yoko Maekawa, Tomoko Tanaka, Etsuji Shimada, and Yoshihiro Kakeji have no conflicts of interest or financial ties to disclose.

\section{References}

1. West NP, Hohenberger W, Weber K, Perrakis A, Finan PJ, Quirke P (2010) Complete mesocolic excision with central vascular ligation produces an oncologically superior specimen compared with standard surgery for carcinoma of the colon. J Clin Oncol 28(2):272-278. doi:10.1200/JCO.2009.24.1448

2. Park IJ, Choi GS, Kang BM, Lim KH, Jun SH (2009) Lymph node metastasis patterns in right-sided colon cancers: is segmental resection of these tumors oncologically safe? Ann Surg Oncol 16(6):1501-1506. doi:10.1245/s10434-009-0368-x 